\title{
High frequency quasi-normal modes for black holes with generic singularities II: Asymptotically non-flat spacetimes
}

\author{
Archisman Ghosh* \\ Department of Physics, Indian Institute of Technology Kanpur, \\ Kanpur-208016, India. \\ S. Shankaranarayanan ${ }^{\dagger}$ \\ HEP Group, The Abdus Salam International Centre for Theoretical Physics, \\ Strada costiera 11, 34100 Trieste, Italy. \\ Saurya Das ${ }^{\ddagger}$ \\ Department of Physics, University of Lethbridge, \\ 4401 University Drive, Lethbridge, Alberta T1K 3M4, Canada
}

(Dated: October 1, 2018)

\begin{abstract}
The possibility that the asymptotic quasi-normal mode (QNM) frequencies can be used to obtain the Bekenstein-Hawking entropy for the Schwarzschild black hole - commonly referred to as Hod's conjecture - has received considerable attention. To test this conjecture, using monodromy technique, attempts have been made to analytically compute the asymptotic frequencies for a large class of black hole spacetimes. In an earlier work, two of the current authors computed the high frequency QNMs for scalar perturbations of $(D+2)$-dimensional spherically symmetric, asymptotically flat, single horizon spacetimes with generic power-law singularities. In this work, we extend these results to asymptotically non-flat spacetimes. Unlike the earlier analyses, we treat asymptotically flat and de Sitter spacetimes in a unified manner, while the asymptotic anti-de Sitter spacetimes is considered separately. We obtain master equations for the asymptotic QNM frequency for all the three cases. We show that for all the three cases, the real part of the asymptotic QNM frequency in general - is not proportional to $\ln (3)$ thus indicating that the Hod's conjecture may be restrictive.
\end{abstract}

PACS numbers: 04.30.-w,04.60.-m,04.70.-s,04.70.Dy

\section{INTRODUCTION}

Classical, damped perturbations about a fixed background which propagate to spatial infinity are commonly referred to as quasi-normal modes (hereafter QNMs) (for excellent reviews, see Refs. [1,2]). In general, for a gravitating object like a star, QNM frequencies depend on (i) the properties of the perturbation such as, the source of the perturbation, the origin of perturbation, the duration of the perturbation etc. and (ii) intrinsic properties of the gravitating object. However, for the black hole spacetimes, the real (which corresponds to the frequency of the oscillation) and complex part (which corresponds to the damping rate) of the QNM frequencies are independent of the initial perturbations and thereby characterize the black hole completely. Due to this property, over the last three decades, the black hole QNM frequencies have attracted a considerable amount of attention.

Although QNMs are purely classical and have no quantum mechanical origin, there have been indications from two different fronts - that these carry some information about quantum gravity [3-6]. More specifically, it

\footnotetext{
*E-mail:archis@iitk.ac.in

${ }^{\dagger}$ E-mail:shanki@ictp.trieste.it

${ }_{\text {E-mail : saurya.das@uleth.ca }}$
}

has been shown that QNM can be a useful tool in understanding the AdS/CFT correspondence. In other words, it has been shown that there is a one-to-one mapping of the damping time scales (evaluated via simple QNM techniques) of black holes in Anti-de Sitter spacetimes and the thermalization time scales of the corresponding conformal field theory (which are, in general, difficult to compute) $[3,4]$. However, the primary reason for the recent interest in QNM comes from its connection to the black hole entropy $[5,6]$.

Based on Nollert's numerical result [7], Hod conjectured that the real part of the asymptotic QNM frequency should be treated as the characteristic transition frequency of a Schwarzschild black hole [5]. Using this conjecture and Bekenstein's conjecture - the black hole area must be quantized [8-10] - he obtained the Bekenstein-Hawking entropy for the Schwarzschild black hole. He also showed that this approach is compatible with the statistical mechanical interpretation of black hole entropy. Later, Dreyer [6] reconciled Hod's result with the loop quantum gravity calculation for the Bekenstein-Hawking entropy (for criticism of this result, see Refs. [11-14]).

Nollert's analytical result was confirmed analytically by two different methods $[15,16]$. In Ref. [15], the author used Nollert's continued fraction expansion for the 4-dimensional Schwarzschild and showed that the asymp- 
totic QNM frequencies are given by the following relation:

$$
\omega_{\mathrm{QNM}}=2 \pi i T_{H}\left(n+\frac{1}{2}\right)+T_{H} \ln (3)+\mathcal{O}\left(n^{-1 / 2}\right),
$$

where $n$ is an integer and $T_{H}$ is the Hawking temperature of the Schwarzschild black hole. In Ref. [16], using the monodromy technique, the authors confirmed Nollert's result and showed that Eq. (1) also holds true for $D$ dimensional Schwarzschild spacetime. These provided tremendous impetus to verify Hod's conjecture for a large class of black hole spacetimes. (For a partial list of references, see [17-44].)

Hod's conjecture rests heavily on the fact that the real part of the asymptotic QNM frequencies is proportional to the logarithm of an integer. The natural question which has lead to a considerable amount of attention in the field is the following: Is Hod's conjecture universally valid for all black hole spacetimes? In an attempt to address this question, two of the current authors computed high frequency quasi-normal frequencies for a single-horizon general spherically symmetric spacetimes with generic singularities and near-horizon properties [38] (hereafter referred to as I). For these spacetimes, using the monodromy approach, a master equation for the asymptotic QNM frequency was obtained. It was also shown that the real part of the high frequency QNM has a logarithmic dependence whose argument need not necessarily be an integer. However, the result rests on the assumption of asymptotic flatness. In this work, we extend the analysis for asymptotically non-flat - de Sitter and anti-de Sitter - spacetimes.

There have been attempts in the literature to obtain high QNM frequencies for asymptotically non-flat spacetimes [39, 40]. Recently, Natario and Schiappa [40] have done a detailed studied of $(D+2)$-dimensional Schwarzschild de Sitter and Anti-de Sitter spacetimes. Our treatment differs from that of Natario and Schiappa's analysis [40] in two ways: (i) As mentioned earlier, we do not assume any form of the metric except at the event-horizon and at the origin. At spatial infinity, we assume that the spacetime is asymptotically flat, de Sitter or Anti-de Sitter. (ii) Broadly, the numerical results for the asymptotically flat, de Sitter and Anti-de Sitter spacetimes suggest two classes of high frequency QNMs (see Sec. (IV A) for more details). We demonstrate that the two class of high frequency QNMs can be related to the two class of boundary conditions (see Secs. (V,VI) for more details). Thus, unlike the earlier analyses, we treat asymptotically flat and de Sitter spacetimes in a unified manner, while the asymptotic anti-de Sitter spacetime is considered separately.

The main results of the paper are as follows: (i) We obtain the master equations for the asymptotic QNM frequency for all the three cases [see Eqs. $(64,65,74)$ ]. (ii) We show that for all the three cases, the real part of the asymptotic QNM frequency, in general, is not proportional to $\ln (n)$ [where $n$ is an integer] thus indicating that the Hod's conjecture may not be valid for large class of black hole spacetimes. (iii) We show that, for all the three cases, the high QNM frequencies have an universal feature. The high QNM frequencies depend on the parameters of the metric in a specific manner i. e. $(D q-2) / 2$ [see Sec. (VI) for more details].

The rest of the paper is organized as follows. In the next section, we briefly discuss generic properties of the spacetime near the horizon(s), singularity and spatial infinity. In Sec. (III), we briefly discuss the scalar perturbations in the general spherically symmetric backgrounds and the boundary conditions for the asymptotic flat, de Sitter and anti-de Sitter spacetimes. In Sec. (IV), we discuss the numerical results for the asymptotically (non)flat spacetimes and the key properties of the monodromy technique. In Sec. (V), we discuss the Stokes lines, contours and boundary conditions for all the three cases. In Sec. (VI), we obtain the asymptotic QNM frequencies for all the three cases. In Sec. (VII), we apply our general results to specific black holes. Finally, we conclude in Sec. (VIII) summarizing our results.

\section{SPHERICALLY SYMMETRIC BLACK HOLE}

In this section, we briefly review the key properties of spherically symmetric spacetime. (For more details, we refer the readers to $\mathbf{I}$.) The line-element for an interval in a $(D+2)$-dimensional spherically symmetric spacetime $(\mathcal{M}, g)$ (with a boundary $\partial \mathcal{M})$ is

$$
\begin{aligned}
d s^{2} & =-f(r) d t^{2}+\frac{d r^{2}}{g(r)}+\rho^{2}(r) d \Omega_{D}^{2}, \\
& =f(r)\left[-d t^{2}+d x^{2}\right]+\rho^{2}(r) d \Omega_{D}^{2},
\end{aligned}
$$

where $f(r), g(r)$ and $\rho(r)$ are arbitrary (continuous, differentiable) functions of the radial coordinate $r, d \Omega_{D}^{2}$ is the metric on the unit $S^{D}$ and

$$
x=\int \frac{d r}{\sqrt{f(r) g(r)}},
$$

is commonly referred to as tortoise coordinate. The lineelement (3) factorizes into the product of two spaces $\mathcal{M}^{2} \times S^{D}$, where $\mathcal{M}^{2}$ is the 2 -dimensional spacetime with Minkowskian topology.

As in $\mathbf{I}$, to keep the discussion general, we do not assume any form for $f(r), g(r)$ and $\rho(r)$. However, we assume the following generic properties of the spacetime: (i) The spacetime has a singularity (say, at $r=0$ ). Near the singularity, we assume that the line-element is given by Szekeres-Iyer [45, 46] metric viz. (5) below. (ii) The spacetime has one event horizon (say at, $r=r_{h}$ ). Near the event horizon, we assume that the line-element takes the form of Rindler metric. (iii) Towards the spatial infinity (say, as $r \rightarrow \infty$ ), we assume that the spacetime is flat, de Sitter or Anti-de Sitter. In the rest of the section, we discuss the spacetime properties in these regions. 


\section{A. Generic singularity and horizon structure}

Near the singularity $(r \rightarrow 0)$, Szekeres-Iyer [45, 46] (see also, Ref. [47]) had shown that a large class of spherically symmetric black holes (2) take the following form:

$$
\begin{aligned}
d s^{2} \stackrel{r \rightarrow 0}{\approx} \eta r^{\frac{2 p}{q}} d y^{2}-\frac{4 \eta}{q^{2}} r^{\frac{2(p-q+2)}{q}} d r^{2}+r^{2} d \Omega_{D}^{2} \\
\quad=\eta x^{p}\left(d y^{2}-d x^{2}\right)+x^{q} d \Omega_{D}^{2}
\end{aligned}
$$

where $\eta= \pm 1,0$ corresponding to the space, time, nulllike singularities and $p, q$ are the power-law indices which are purely real. Comparing Eqs. $(2,5)$, we have

$$
f(r)=-\eta \beta^{2} r^{\frac{2 p}{q}} ; \frac{1}{g(r)}=-\frac{4 \eta}{q^{2}} r^{\frac{2(p-q+2)}{q}} ; \rho(r)=r,
$$

and the corresponding tortoise coordinate is

$$
x \sim \int \frac{d r}{r^{1-\frac{2}{q}}}=r^{\frac{2}{q}} .
$$

It is interesting to note that $x$ depends only on $q$ and not on $p$.

Near the horizon, we assume that the general spherically symmetric line-element (2) takes the form of Rindler metric:

$$
d s^{2} \rightarrow-\kappa_{h}^{2} \gamma^{2} d t^{2}+d \gamma^{2}+\rho^{2}\left(r_{h}\right) d \Omega_{D}^{2}
$$

where

$$
\gamma=\frac{1}{\kappa_{h}} \sqrt{f}, \frac{d \gamma}{d r}=\frac{1}{2 \kappa_{h}} \frac{d_{r} f}{\sqrt{f}}, \kappa_{h}=\frac{1}{2}\left(\sqrt{\frac{g(r)}{f(r)}} d_{r} f\right)_{r_{h}}
$$

and the tortoise coordinate is given by

$$
x \sim \frac{1}{2 \kappa_{h}} \ln \left(r-r_{h}\right)
$$

\section{B. Spatial Infinity}

In this sub-section, we discuss spatial asymptotic properties for the three cases - asymptotically flat, de Sitter and Anti-de Sitter spacetimes.

\section{Asymptotic flat spacetimes:}

A spacetime $(\mathcal{M}, g)$ is said to be asymptotically flat if it is asymptotically empty, i. e. $R_{\mu \nu}=0$ in an open neighborhood of $\partial \mathcal{M}$ in $\mathcal{M}$. A static observer in these spacetimes is bounded by the past/future event horizons and $\mathcal{I}^{ \pm}$.

The line-element (2) towards the spatial infinity takes the following form:

$$
d s^{2} \simeq-d t^{2}+d r^{2}+r^{2} d \Omega_{D}^{2} .
$$

Comparing Eqs. (11, 2), we have

$$
f(r)=g(r) \sim 1 ; \rho(r) \sim r ; x \sim r .
$$

For these spacetimes, from our assumption, there exists only one physical (real, positive) horizon at $r=r_{h}$ whose surface gravity is given by the relation (9).

\section{Asymptotic de Sitter spacetimes:}

A static observer is bounded by the past/future event horizons and past/future cosmological horizons. Although the coordinate $r$ goes up to $\infty$, the physical region terminates at the cosmological horizon $\left(r=r_{c}, x \rightarrow \infty\right)$. For computation of conserved charges in asymptotically de Sitter spacetimes, see for instance, Ref. [48].

The line-element (2) at spatial infinity takes the following form:

$$
d s^{2} \simeq \frac{r^{2}}{\ell^{2}} d t^{2}-\frac{\ell^{2}}{r^{2}} d r^{2}+r^{2} d \Omega_{D}^{2}
$$

where $\ell^{2}$ is related to the $(D+2)$-dimensional positive cosmological constant, i. e.,

$$
\Lambda=\frac{D(D+1)}{2 \ell^{2}} .
$$

Comparing Eqs. (2,13), we have

$$
f(r)=g(r) \sim-\frac{r^{2}}{\ell^{2}} ; \rho(r) \sim r ; x \sim x_{0}+\frac{\ell^{2}}{r} .
$$

In this case, unlike the previous two cases, the spacetime contains two physical horizons - event horizon $\left(r=r_{h}\right)$ and cosmological horizon (say, at $r=r_{c}$ ). The surface gravity at $r_{h}$ is given by (9). The surface gravity of the cosmological horizon is

$$
\kappa_{c} \equiv \frac{1}{2}\left(\sqrt{\frac{g(r)}{f(r)}} \frac{d f(r)}{d r}\right)_{r=r_{c}},
$$

which we set to be negative.

\section{Asymptotic Anti-de Sitter spacetimes:}

A static observer in these spacetimes is bounded by the past/future event horizons and the finite spatial boundary. For computation of conserved charges in asymptotically anti-de Sitter spacetimes, see Ref. [49, 50].

The line-element (2) near the spatial infinity takes the following form:

$$
d s^{2} \simeq-\frac{r^{2}}{\ell^{2}} d t^{2}+\frac{\ell^{2}}{r^{2}} d r^{2}+r^{2} d \Omega_{D}^{2}
$$

where $\ell^{2}$ is related to the $(D+2)$-dimensional negative cosmological constant, i. e.,

$$
\Lambda=-\frac{D(D+1)}{2 \ell^{2}} .
$$


Comparing Eqs. $(2,17)$, we have

$$
f(r)=g(r) \sim \frac{r^{2}}{\ell^{2}} ; \rho(r) \sim r ; x \sim x_{0}-\frac{\ell^{2}}{r},
$$

where $x_{0}$ is asymptotic value of $x$ and, in general, depends on the negative cosmological constant and black hole properties. For these spacetimes, as-well, there exists only one physical horizon, at $r=r_{h}$ with surface gravity $\kappa_{h}$ as in (9).

\section{QUASI-NORMAL MODES}

In this section, we obtain the differential equation corresponding to scalar field propagating in $(D+$ 2 )-dimensional spherically symmetric spacetime (2) and discuss the "canonical" boundary conditions corresponding to the three cases. [By canonical, we mean the boundary conditions applied in the real $x$ i.e. in the range $(-\infty, \infty)$.]

\section{A. Scalar perturbations}

The perturbations of a $(D+2)$-dimensional static black holes (2) can result in three kinds - scalar, vector and tensor - of gravitational perturbations (see for example, Ref. [51]). The higher dimensional tensor perturbations, which is of our interest in this work, correspond to the well-known four-dimensional Regge-Wheeler potential $^{1}$. The evolution equation for these perturbations correspond to the equation of motion of the massless, minimally coupled scalar field, i. e.,

$$
\square \Phi \equiv \frac{1}{\sqrt{-g}} \partial_{\mu}\left(\sqrt{-g} g^{\mu \nu} \partial_{\nu} \Phi\right)=0 .
$$

The symmetry of the line-element (2) allows us to decompose the scalar field modes as:

$$
\Phi\left(x^{\mu}\right)=\rho(r)^{-\frac{D}{2}} R(r) e^{i \omega t} Y_{l m_{1} \ldots m_{D-1}},
$$

where $Y_{l m_{1} . . m_{D-1}}$ are Hyper-spherical harmonics and the function $R(r)$ satisfies the differential equation

$$
\frac{d^{2} R(r)}{d x^{2}}+\left[\omega^{2}-V(r)\right] R(r)=0
$$

\footnotetext{
${ }^{1}$ Note that we loosely refer to these perturbations as scalar. This is due to the fact that there is a one-to-one correspondence between the massless scalar field propagating in the fixed background and the tensor perturbation equations derived from the linear perturbation theory.
}

where $x$ is given by (4), $r$ is understood to be $r(x)$ and

$$
\begin{aligned}
V(r) & =\frac{l(l+D-1)}{\rho^{2}(r)} f(r)+\left(\frac{D}{2}\right) \rho(r)^{-\frac{D}{2}} \sqrt{f(r) g(r)} \\
& \times \frac{d}{d r}\left\{\rho(r)^{\frac{D-2}{2}} \frac{d \rho(r)}{d r} \sqrt{f(r) g(r)}\right\},
\end{aligned}
$$

is the generalized Regge-Wheeler potential. Before discussing the boundary conditions, it is important to know the structure of the singularities of the differential equation at the three $-0, r_{h}, r_{c}($ or $\infty)$ - points. For the differential equation (22), we have: (i) $r=r_{h}$ is a regular singular point. (ii) $r \rightarrow \infty$ (relevant for the asymptotically flat and Anti-de Sitter spacetimes) is an irregular singular point. $r=r_{c}$ is a regular singular point. (iii) In order for $r=0$ to be a regular singular point it can be easily shown that $p, q$ must satisfy the following conditions (see $\mathbf{I}$ ):

$$
q>0 \text { and } p-q+2>0 .
$$

\section{B. Canonical boundary conditions}

QNMs are solutions to the differential equation (22) subject to a specific (physically motivated) boundary conditions $^{2}$. The Wronskian of these modes vanish which gives the corresponding QNM frequencies [2].

In order to obtain the QNM frequencies corresponding to the differential equation (22), we need to know the mode functions. The Regge-Wheeler potential is a complicated function of $f(r), g(r)$ and $\rho(r)$. Hence, in general, the differential equation (22) can not be solved exactly. In such a situation, asymptotic analysis is a useful tool to extract some physical information. In the case of QNM, the asymptotic analysis also provides us with the identification of the boundary conditions.

\section{Asymptotic flat spacetimes:}

The generalized Regge-Wheeler potential decays exponentially near the event-horizon and as a power-law near spatial infinity, i.e.,

$$
V[r(x)] \stackrel{x \rightarrow-\infty}{\simeq} \exp \left(2 \kappa_{h} x\right) ; V[r(x)] \stackrel{x \rightarrow \infty}{\simeq} \frac{1}{x^{2}} .
$$

Thus, the general solution to Eq. (22) near the two boundary points is a superposition of plane-waves:

$$
R[x] \stackrel{x \rightarrow \pm \infty}{\sim} C_{1}^{ \pm} \exp (i \omega x)+C_{2}^{ \pm} \exp (-i \omega x),
$$

where $C_{1}^{ \pm}, C_{2}^{ \pm}$are the constants determined by the choice of the boundary conditions. QNM boundary condition

\footnotetext{
2 Note that, we choose the sign of the exponent $e^{i \omega t}$ in equation (21) above, we fix the sign $\Im(\omega)>0$. This is because $\Im(\omega)<0$ leads to solutions growing with time, which are unphysical.
} 
corresponds to

$$
C_{2}^{-}=0 ; C_{1}^{+}=0 \Longrightarrow R(x) \sim e^{ \pm i \omega x} \text { as } x \rightarrow \mp \infty .
$$

Physically, the boundary conditions mean that no classical radiation emerge from the (future) event horizon, and no radiation originates at spatial infinity.

\section{Asymptotic de Sitter spacetimes:}

The generalized Regge-Wheeler potential decays exponentially near the two - event and cosmological - horizons i. e.,

$$
V[r(x)] \stackrel{x \rightarrow-\infty}{\simeq} \exp \left(2 \kappa_{h} x\right) ; V[r(x)] \stackrel{x \rightarrow \infty}{\simeq} \exp \left(-2\left|\kappa_{c}\right| x\right) .
$$

As in the case of asymptotically flat spacetime, the solution near the two boundary points is a superposition of plane-waves (26) and hence, the boundary conditions are same as that of asymptotically flat space (27).

Physically, the boundary conditions mean that no classical radiation emerge from the event and cosmological horizons.

\section{Asymptotic anti-de Sitter spacetimes:}

The generalized Regge-Wheeler potential decays exponentially in the event horizon, however the potential grows at spatial infinity:

$$
V[r(x)] \stackrel{x \rightarrow-\infty}{\simeq} \exp \left(2 \kappa_{h} x\right) ; V[r(x)] \stackrel{x \rightarrow x_{0}}{\simeq} \frac{j_{\infty}^{2}-1}{4\left(x-x_{0}\right)^{2}}
$$

where at spatial infinity $x$ goes as

$$
x \sim x_{0}-\frac{\ell^{2}}{r} \quad \text { and } \quad j_{\infty}=D+1 .
$$

[Even though, this is a standard result and can be found in other references (see, for instance, Ref. [52]), we have given the relevant steps in Appendix (A) for completeness.] Thus, the general solution to Eq. (22) near the two boundary points is given by

$$
\begin{aligned}
& R[r(x)] \stackrel{x \rightarrow-\infty}{\underset{\simeq}{\simeq}} C_{1}^{-} \exp (i \omega x)+C_{2}^{-} \exp (-i \omega x) \\
& \stackrel{x}{\simeq} C_{1}^{+}\left(x_{0}-x\right)^{-\frac{D}{2}}+C_{2}^{+}\left(x_{0}-x\right)^{\frac{D}{2}+1}
\end{aligned}
$$

QNM boundary condition corresponds to

$$
\begin{aligned}
& C_{2}^{-}=0 \Longrightarrow R(x) \sim e^{i \omega x} \quad \text { as } \quad x \rightarrow-\infty \\
& C_{1}^{+}=0 \Longrightarrow R(x) \simeq 0 \quad \text { as } \quad x \rightarrow x_{0} .
\end{aligned}
$$

Physically, the boundary conditions mean that no classical radiation emerge from the (future) event horizon, and modes reflect at the spatial boundary. It is necessary to have a reflecting boundary conditions for the following reasons: (i) The perturbation equation (22) is obtained from the first order perturbation theory implying that the stress-tensor of the perturbation is small compared to the background. If $C_{1}^{+} \neq 0$, then this assumption is violated and leads to inconsistency. (ii) To the linear order, the perturbations conserve energy-momentum. The exponential growth of the modes would violate energy conservation [53].

\section{NUMERICAL RESULTS AND MONODROMY TECHNIQUE}

In this section, we briefly discuss the numerical results of the asymptotic QNM frequencies. We also briefly discuss the monodromy technique which has proven to be useful to analytically calculate asymptotic QNM frequencies.

\section{A. Numerical results}

As mentioned earlier, the perturbation equation (22) cannot be solved exactly and one has to resort to approximation methods to obtain analytical results for QNM frequencies. Broadly, the analytical/numerical approaches in obtaining the QNM frequencies can be classified into four categories: (i) Approximating the Regge-Wheeler potential with some simple functions to obtain the exact QNM frequencies. (ii) Solving the perturbation equation iteratively by using the well-known techniques like WKB or Born approximation (iii) Continued fraction technique. (iv) Monodromy technique. (For an excellent review of the above techniques, see Ref. [1].) It is needless to say that nearly all of these approaches have their own limitations; certain analytical techniques are useful in certain QNM frequency range while certain others techniques for certain other ranges. For instance, the monodromy technique - which is of our interest in this work - has proven to be useful for obtaining asymptotic QNM frequencies.

The numerical results for asymptotic QNM frequencies have been obtained by various authors following Nollert's seminal result [7] for 4-dimensional Schwarzschild. Nollert's results have been extended to other dimensions by Cardoso and his collaborators $[25,54]$ (see also Ref. [55]). In the case of asymptotic AdS spacetimes, the first numerical calculation was done by Horowitz and Hubeny [4] for 4,5 and 7-dimensional Schwarzschild-AdS black holes in the large black hole limit. Their results have been extended by host of other authors for large, intermediate and small black hole limits $[24,26,56,57]$. For the asymptotic dS spacetimes, the numerical results are obtained for Schwarzschild-de Sitter spacetime by various authors [58-61].

Broadly, two classes have emerged from these numerical results:

1. For the asymptotically flat and de Sitter spacetimes, the numerical results for the high-frequency 
QNMs indicate that

$$
\Im\left(\omega_{Q N M}\right) \gg \Re\left(\omega_{Q N M}\right) .
$$

2. For the asymptotically Anti-de Sitter spacetimes, the numerical results for the high-frequency QNMs indicate that

$$
\Im\left(\omega_{Q N M}\right) \sim \Re\left(\omega_{Q N M}\right) .
$$

In the previous section, we showed that the QNM boundary conditions for the asymptotic flat and de Sitter spacetimes are identical, however, it is different in the case of asymptotic Anti-de Sitter spacetimes. Using the numerical results and the boundary condition, it is easy to note the following: The structure of the asymptotic QNM frequencies crucially depend on the choice of the boundary conditions.

In Sec. (VI), we obtain the high QNM frequencies for the three cases. Unlike the earlier analyses, we treat asymptotically flat and de Sitter spacetimes in a unified manner, while the asymptotically anti-de Sitter spacetime is considered separately.

\section{B. Monodromy technique}

QNMs are damped modes whose frequencies are complex. This implies that the QNM frequencies will have positive imaginary parts. It follows that each QNM eigenfunction $\exp \left(i \omega_{n} t\right) R[r(x)]$ (for instance, in the asymptotically flat spacetime) will grow/decay exponentially both towards infinity and at the horizon. Thus, as the QNM mode traces from the horizon to infinity the modes can grow exponentially within a small region. In other words, the modes which are exponentially suppressed in a region can grow exponentially in the nearby region $^{3}$. This implies that analytical/numerical techniques require exponential precision. Monodromy technique has proved to be a powerful and flexible approach in obtaining the high frequency QNMs.

Monodromy technique has five key steps:

\section{Analytically continue the QNMs in the complex $r$} (or x) plane.

This allows one to study the properties of these modes near the singularity $(r=0)$. In the monodromy technique, unlike other techniques, both $\omega$ and $x$ are complex.

2. Map the QNMs from the real $x$ to the complex $\omega x$ plane.

\footnotetext{
3 A curve which separates these two regions is referred to as ramification line and the two regions separated by the ramification line are called ramification regions. In the language of complex analysis, these ramification lines are nothing but the branch cuts.
}

Mathematically, this involves finding QNM solutions to the following differential equation

$$
\frac{d^{2} R(r)}{d(\omega x)^{2}}+\left[1-\frac{V(r)}{\omega^{2}}\right] R(r)=0 .
$$

Even though, Eqs. $(22,36)$ look identical, operationally they are quite different: Firstly, $\omega$ is a complex number and hence the independent variable $\omega x$ is complex even if $x$ is purely real. Secondly, since the independent variable $(\omega x)$ is complex, the issues of the existence, uniqueness of solutions [to the differential equation (36)] satisfying the boundary conditions is non-trivial compared to that of Eq. (22).

In the large asymptotic limit of $|\omega|$, Eq. (36) can be approximated to

$\frac{d^{2} R(r)}{d(\omega x)^{2}}=-R(r) \Longrightarrow R[\omega x] \sim \exp ( \pm i \omega x)$.

Thus, in the high-frequency limit, QNMs can be approximated to be the superposition of plane-waves in whole of complex $\omega x$ plane except at the isolated singularities or branch cuts .

Setting $\omega=\omega_{R}+i \omega_{I}, x=x_{R}+i x_{I}$, the asymptotic modes (26) take the following form:

$$
\begin{aligned}
R[\omega x] & \sim \exp \left[ \pm i\left(\omega_{R} x_{R}-\omega_{I} x_{I}\right)\right] \\
& \times \exp \left[\mp\left(\omega_{R} x_{I}+\omega_{I} x_{R}\right)\right] .
\end{aligned}
$$

3. Identify the Stokes line, contours in the $\omega x$ complex plane.

The Stokes lines are defined by the condition $\Im(\omega x)=0{ }^{4}$. Under the condition, Eq. (38) gets simplified to

$$
R[\omega x] \sim \exp \left[ \pm i\left(\omega_{R} x_{R}-\omega_{I} x_{I}\right)\right]
$$

Thus, all along the Stokes line the modes are oscillating without any exponentially growing/decaying solutions. In the next section, we will show that even near the singularity the solutions to Eq. (36) are plane waves.

4. Use the numerical results for the high-frequency QNMs to translate the condition for the Stokes line in the $\omega x$ complex plane to a condition in the complex $x$ plane ${ }^{5}$.

\footnotetext{
4 The Stokes lines are multi-valued near the horizon where the function $x$ is multi-valued.

${ }^{5}$ It may be worth noting that the monodromy technique requires input about the behavior of the asymptotic frequencies from the numerical analyses. Without the numerical results, it is not possible to analyze the Stokes line in the $\omega x$ plane.
} 
For the asymptotically flat spacetime, the numerical results for the asymptotic QNM frequencies indicate that $\Im(\omega) \gg \Re(\omega)$. Thus the condition for the Stokes line translates to $\Re(x) \simeq 0$ which is nothing but the Anti-Stokes line in the complex $x$ manifold.

5. Identify a closed contour in the complex $x$ plane and calculate the monodromy.

Calculating the monodromy gives the analytical expression for the the asymptotic QNM frequencies.

\section{STOKES LINE, CONTOURS AND MONODROMY BOUNDARY CONDITIONS}

In the previous section, we discussed main features of the monodromy technique. In this section, we obtain the Regge-Wheeler potential in the three regimes - singularity, horizon(s) and spatial infinity - and discuss generic properties of the Stokes line, contours and monodromy boundary conditions for the three cases. [We refer to the conditions in the $\omega x$ plane as "monodromy" boundary conditions.]

\section{A. Regge-Wheeler potential}

Near the singularity, the asymptotic properties of the spacetimes will not play any role. Hence, for the all the three cases, the singularity structure will be identical. As mentioned earlier, we assume that near the singularity the line-element is given by Szekeres-Iyer metric. The generalized Regge-Wheeler potential near the singularity is

$$
\begin{aligned}
& V[r(x)] \stackrel{r \rightarrow 0}{\sim} \frac{q D}{8}\left(\frac{q D}{2}-2\right) r^{-\frac{4}{q}}=\frac{\left(\frac{q D}{2}-1\right)^{2}-1}{4 x^{2}} \\
& \equiv \frac{j^{2}-1}{4 x^{2}},
\end{aligned}
$$

where

$$
j=\frac{q D}{2}-1
$$

Substituting the potential in Eq. (22), we get (cf. Ref. [62], p. 362)

$$
R(x) \sim A_{+} \sqrt{2 \pi \omega x} J_{\frac{j}{2}}(\omega x)+A_{-} \sqrt{2 \pi \omega x} J_{-\frac{j}{2}}(\omega x),
$$

where the quantities $J_{\mu}$ are the Bessel functions of order $\mu$. Using the asymptotic behavior of the Bessel functions (cf. Ref. [62], p. 364)

$$
\lim _{|z| \rightarrow \infty} J_{\nu}(z)=\sqrt{\frac{2}{\pi z}} \cos \left(z-\frac{1}{2} \nu \pi-\frac{1}{4} \pi\right),
$$

we get

$$
\sqrt{2 \pi \omega x} J_{ \pm \frac{j}{2}}(\omega x) \sim 2 \cos \left(\omega x-\alpha_{ \pm}\right)
$$

where

$$
\alpha_{ \pm}=\frac{\pi}{4}(1 \pm j) .
$$

Thus, the asymptotic form of $R$ is

$$
\begin{aligned}
R(x) & \sim\left(A_{+} e^{i \alpha_{+}}+A_{-} e^{i \alpha_{-}}\right) e^{-i \omega x} \\
& +\left(A_{+} e^{-i \alpha_{+}}+A_{-} e^{-i \alpha_{-}}\right) e^{i \omega x} .
\end{aligned}
$$

Near the horizons, the Regge-Wheeler potential decays exponentially [cf. Eqs. (25,28)]. Hence, the mode function $R(x)$ is a superposition of plane waves.

Near the spatial infinity, the potential decays (grows) for the asymptotically flat (anti-de Sitter) spacetimes. Hence, the mode function $R(x)$ is a superposition of plane waves (exponentially decaying/growing solutions).

\section{B. Stokes line}

In the monodromy technique, unlike the canonical techniques, we need to obtain solution to the differential equation (36) in the complex plane. Thus, the canonical boundary conditions which were defined on the boundary points in the $x$-line have to be redefined in the $\omega x$ complex. In other words, the canonical boundary conditions (in $x$ line) need to be mapped to the boundary conditions on the $\omega x$ curve.

There is no unique choice for the $\omega x$ curve. In this work, we will assume $\omega x$ to be along the Stokes line i. e. $\Im(\omega x)=0$. There are couple of reasons for this choice: Firstly, all along this curve the QNM solutions $\left(e^{ \pm i \omega x}\right)$ are purely oscillating and do not contain any exponentially growing/decaying modes. Secondly, using the numerical results of the asymptotic QNM frequencies, the Stokes-line condition in the $\omega x$ plane can be transformed onto a condition in the $x$ plane. In the case of asymptotically flat and de Sitter cases, the Stokes line condition translates to $\Re(x) \simeq 0$ [16] while for asymptotically antide Sitter spacetimes, the Stokes line condition translates to $\Im(\exp [i \pi / 3] x)=0[63]$.

In the rest of this subsection, we discuss the generic properties of the Stokes line near the singularity and asymptotic infinity.

\section{Near the generic singularity:}

Using the relation (7), between $x$ and $r$, near the generic singularity, and setting

$$
r=\rho e^{i \theta} \quad \text { where } \quad \rho, \theta \in \mathcal{R},
$$

the Stokes line condition take the following simple form:

$$
\tan \left(\frac{2 \theta}{q}\right)=-\arg \omega \quad \Longrightarrow \quad \theta=-\frac{q}{2} \tan ^{-1}[\arg \omega]+\frac{n \pi q}{2}
$$


where $n$ is an integer. This implies that (i) Near the generic singularity, the Stokes line have $2 D$ branches and (ii) The angle between adjacent branches are $(\pi q / 2)$.

In order to illustrate these features, we have plotted the Stokes lines for the three cases in Figs. $(1,2,3)$.

\section{Near the spatial infinity/cosmological horizon:}

For the asymptotic flat spacetimes, using the fact that $x \sim r$ (near the spatial infinity), it is easy to see that the Stokes line diverges to infinity.

For the asymptotic de Sitter spacetimes, using the fact that $x \sim 1 / r$ it is easy to see that the Stokes line cross the real axis. Thus, the Stokes line forms a closed contour.

For the asymptotic anti-de Sitter spacetime, using the fact that $x$ is not purely real (in the asymptotic limit) it is easy to see that Stokes line do not cross the real axis. In fact, the angle the Stokes line makes w.r.t the real axis is $\pi / 3^{6}$. It is also easy to see that the Stokes line do not close in this case.

Note that the above arguments are generic and depend only on the asymptotic properties of the spacetime. Further, as noted at the end of Sec. (IV A), the difference in the Stokes lines for the asymptotic de Sitter/flat and anti-de Sitter confirms that the structure of the asymptotic QNM frequencies crucially depend on the choice of the boundary conditions.

\section{Contours and monodromy}

In this subsection, we discuss the choice of contours (to calculate the monodromy) for all the three cases. All along the Stokes lines, the modes are purely oscillating plane-waves without any exponentially growing or decaying solutions. This property of the Stokes-lines is useful to obtain the monodromy around a closed contour for the mode function. Hence, we choose our contours to lie as close as possible to the Stokes line.

\section{Asymptotic flat spacetimes:}

Fig. (1) contains the contour plot for a general $(D+$ 2)-dimensional single-horizon, asymptotically flat spherically symmetric spacetimes. Near the singularity, the Stokes lines have $2 D$ branches. Out of these, two branches $\left(1\right.$ and $\left.1^{\prime}\right)$ of the Stokes line go around the event horizon $\left(r_{h}\right)$ and form a closed contour. Two other branches $\left(2\right.$ and $2^{\prime}$ ) which extend up to infinity do not form a closed contour. [Points $A, A^{\prime}$ correspond to the

\footnotetext{
${ }^{6}$ Using the fact that Stokes line condition can be rewritten as $\Im(\exp [i \pi / 3] x)=0$ and setting $x=x_{0} \exp ( \pm i \theta)$, we get $\theta=$ $\mp \pi / 3$.
}

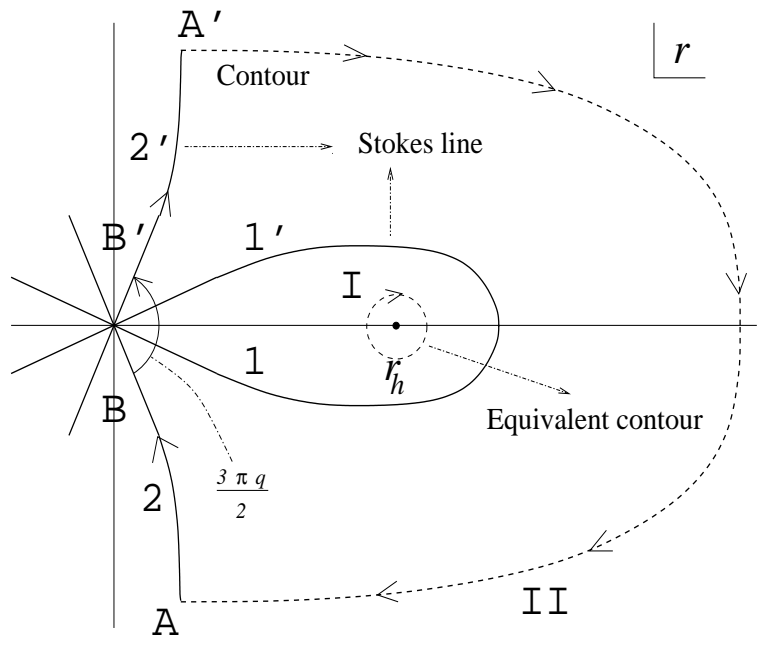

FIG. 1: Stokes lines and contour for asymptotically flat spacetimes.

points at the spatial infinity.] Note that the angle between 2 and $2^{\prime}$ is $(3 \pi q / 2)$.

At the spatial infinity, since the spacetime is flat, the WKB solutions to the differential equation (22) are exact implying that the the mode function is a superposition of plane-waves. In other words, all along the dotted line connecting the points $A$ and $A^{\prime}$ in Fig. (1), the mode functions are superposition of plane-waves.

We compute the monodromy using two equivalent contours $(I, I I)$. The two contours give different contributions to the mode function $R(x)$. While contour $I$ picks up the monodromy contribution from the horizons, contour $I I$ picks up a factor from the generic singularity. Monodromy contribution from contour $I$ is easy to evaluate while that of contour $I I$ is non-trivial and has two terms:

$$
\begin{aligned}
& \text { Monodromy of the mode function } \\
& =\text { Factor by which coeff of } e^{\mp i \omega x} \text { gets multiplied } \\
& \quad \times \text { Monodromy of } e^{\mp i \omega x} .
\end{aligned}
$$

We then equate the monodromies obtained from contours $I$ and $I I$. The steps involved in the calculation are discussed in detail in Sec. (VI).

In either case, due to the logarithmic relation between $r$ and $x$ [cf. Eq. (10)], the $e^{\mp i \omega x}$ parts of the mode function pick up a monodromy. If in the $r$-plane we perform a clockwise rotation around the horizon by $2 \pi$, due to discontinuity across the branch-cut, we get

$$
\begin{aligned}
& \ln \left(r-r_{h}\right) \rightarrow \ln \left(r-r_{h}\right)-2 \pi i \quad \Longrightarrow \quad x \rightarrow x-\frac{\pi i}{\kappa_{h}} \\
& e^{\mp i \omega x} \rightarrow e^{\mp i \omega\left(x-\frac{\pi i}{\kappa_{h}}\right)}=e^{\mp i \omega x} e^{\mp \frac{\pi \omega}{\kappa_{h}}} .
\end{aligned}
$$

Thus, the clock-wise rotation of the plane-wave modes in the equivalent contour will acquire the monodromy of 
the following form:

$$
\text { Monodromy }[\exp (\mp i \omega x)]=\exp \left(\mp \frac{\pi \omega}{\kappa_{h}}\right) \text {. }
$$

\section{Asymptotic de Sitter spacetimes:}

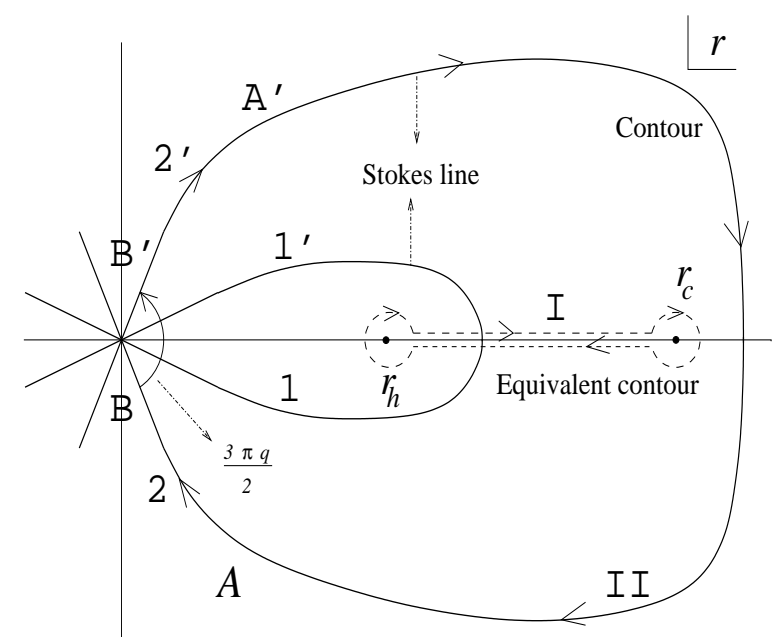

FIG. 2: Stokes lines and contour for asymptotically de Sitter spacetimes.

Fig. (2) contains the contour plot for a general $(D+$ 2)-dimensional single-horizon, asymptotic de Sitter, spherically symmetric spacetimes. As in the asymptotically flat spacetimes, the Stokes lines have $2 D$ branches near the singularity. Out of these, two branches (1 and $\left.1^{\prime}\right)$ of the Stokes line go around the event horizon $\left(r_{h}\right)$ and form a closed contour. Two other branches $(2$ and $2^{\prime}$ ) which extend upto infinity also form a closed contour. Note that, as in the case of asymptotically flat spacetimes, the angle between 2 and $2^{\prime}$ is $(3 \pi q / 2)$. In order to evaluate the monodromy around the contour this is the angle by which we need to deform the contour close to the singularity.

The procedure to compute the monodromy is similar to that of the asymptotically flat spacetime case except that in this case the monodromy has to evaluated for two event and cosmological - horizons. The total monodromy of the mode functions is again given by the relation (49).

Here again, the monodromy contribution from contour $I$ is easy to evaluate while that of contour $I I$ is non-trivial [Eqn. (49)] and we equate the two monodromies from the two contours. The details are discussed in Sec. (VI). In this case, the contours go clockwise around both the horizon and the cosmological horizon. Thus, the planewave modes $e^{\mp i \omega x}$ pick up the following monodromy term

$$
\text { Monodromy }[\exp (\mp i \omega x)]=\exp \left(\mp \frac{\pi \omega}{\kappa_{h}} \mp \frac{\pi \omega}{\kappa_{c}}\right) .
$$

Asymptotic Anti-de Sitter spacetimes:

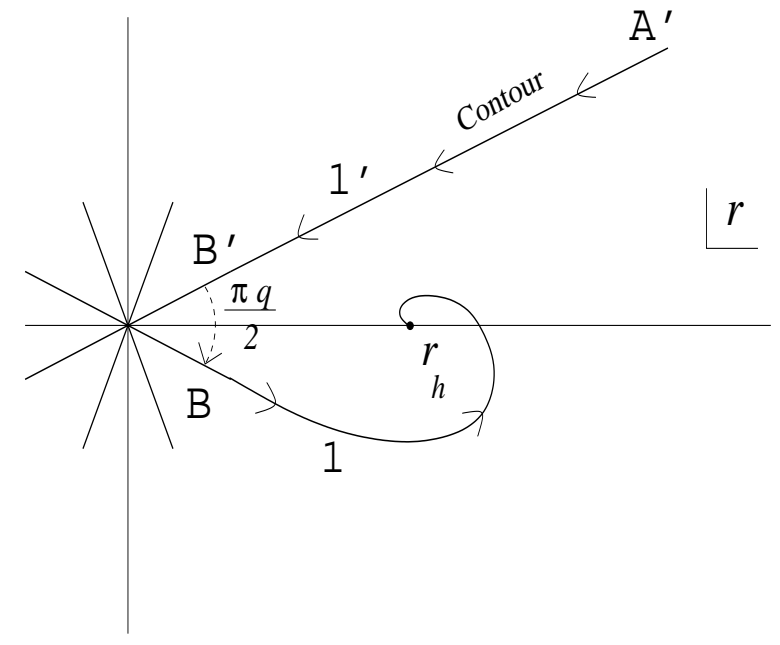

FIG. 3: Stokes lines and contour for asymptotically Anti-de Sitter spacetimes.

Fig. (3) contains the contour plot for a general $(D+$ 2)-dimensional single-horizon, asymptotic anti-de Sitter, spherically symmetric spacetimes. As in the previous two cases, the Stokes lines have $2 D$ branches near the singularity. Utilizing the multi-valuedness of the tortoise coordinate near the horizon, we choose the Stokes line from the horizon to join one branch $\left(1^{\prime}\right)$ of the Stokes line from the origin. Another branch (1), from the origin, is chosen to extend upto infinity.

Unlike the previous two cases, even one of the Stokes line (hence contour) do not close. Note that the angle between 1 and $1^{\prime}$ is $(\pi q / 2)$. This is the angle by which we need to deform the contour close to the singularity.

Since the contour does not close, we can not use the monodromy technique. In this case, we obtain the highfrequency QNM by matching the asymptotic solutions with the exact solutions in the limit of $\omega x-t o \pm \infty$.

\section{Monodromy boundary conditions}

For highly damped modes,

$$
x \rightarrow \pm \infty \quad \Longrightarrow \quad \omega x \rightarrow \pm i \infty
$$

Thus, the boundary points in the real $x$-line are purely imaginary in the $\omega x$ plane [points $A, B$ in Figs. $(1,2)]$. Using the fact that the "canonical" boundary conditions are identical for the asymptotically flat and de Sitter spacetimes and that the contours are similar for the two cases, 
the "monodromy" boundary conditions are given by ${ }^{7}$

$$
R(x) \sim e^{\mp i \omega x} \quad\left\{\begin{array}{ll}
\omega x \rightarrow \pm \infty & \Re(\omega)>0 \\
\omega x \rightarrow \mp \infty & \Re(\omega)<0
\end{array} .\right.
$$

In the case of asymptotic anti-de Sitter spacetimes, it is not possible to map the "canonical" boundary conditions on to the $\omega x$ plane.

\section{COMPUTING THE ASYMPTOTIC QN FREQUENCIES}

In this section, we compute the asymptotic QN frequencies for the general $(D+2)$-dimensional spherically symmetric spacetimes. In the following subsection, using the monodromy technique, we compute the high frequency QNMs for the asymptotically flat and de Sitter spacetimes in a unified manner . In the last subsection, we obtain the high QNM frequencies for the asymptotic anti-de Sitter spacetimes.

\section{A. Asymptotic flat and de Sitter spacetimes}

We first compute the monodromy contribution from contour $I I$. In order to do that, we follow the contour from the negative imaginary axis $(A)$ to the positive imaginary axis $\left(A^{\prime}\right)$ - by passing through the points $B$ and $B^{\prime}$ - and come back to $A$.

The mode function $R(x)$ at $A$ is given by (46). In the case of asymptotically flat spacetimes, as in the previous analyses, it is possible to fix the constants - by applying the boundary conditions (54) - before calculating the monodromy. However, we would like to follow a different procedure: we apply the boundary conditions after calculating the monodromy. In this way, it is possible to obtain the high-frequency QNMs for the asymptotic flat and de Sitter spacetimes in a unified manner.

At $B$, the mode function $R(x)$ is given by (42). To obtain the mode function $R(x)$ at $B^{\prime}$, we need to deform the contour close to the singularity (in the $r$-plane) by an angle $(3 \pi q / 2)$. Using Eq. (7), this translates to a rotation by an angle $(3 \pi q / 2) \times(2 / q)=3 \pi$ in the $x$ plane i.e. $x \rightarrow x^{\prime}=e^{i 3 \pi} x$.

Using the relation

$$
J_{\nu}\left(z e^{i m \pi}\right)=e^{i m \nu \pi} J_{\nu}(z),
$$

we get

$$
\sqrt{\omega x^{\prime}} J_{ \pm \frac{j}{2}}\left(\omega x^{\prime}\right)=e^{i 6 \alpha_{ \pm}} \sqrt{\omega x} J_{ \pm \frac{j}{2}}(\omega x)
$$

\footnotetext{
7 There are some subtleties involved in defining the boundary condition at the spatial infinity. See Ref. [16].
}

Thus, the mode function $R(x)$ at $B^{\prime}$ is given by

$$
\begin{aligned}
R(x) & \sim A_{+} \sqrt{2 \pi \omega x} \exp \left(i 6 \alpha_{+}\right) J_{\frac{j}{2}}(\omega x) \\
& +A_{-} \sqrt{2 \pi \omega x} \exp \left(i 6 \alpha_{-}\right) J_{-\frac{j}{2}}(\omega x) .
\end{aligned}
$$

Using the asymptotic expansion (43), the mode function at $A^{\prime}$ is given by

$$
\begin{aligned}
R(x) & \sim\left(A_{+} e^{5 i \alpha_{+}}+A_{-} e^{5 i \alpha_{-}}\right) e^{-i \omega x} \\
& +\left(A_{+} e^{7 i \alpha_{+}}+A_{-} e^{7 i \alpha_{-}}\right) e^{i \omega x} .
\end{aligned}
$$

As we go along the closed contour from $B$ to $A$, the "new" mode function (58) is different compared to the original mode function (46). The coefficients of $e^{\mp i \omega x}$ in the mode function $R(x)$ are different by a factor

$$
\frac{A_{+} e^{5 i \alpha_{+}}+A_{-} e^{5 i \alpha_{-}}}{A_{+} e^{i \alpha_{+}}+A_{-} e^{i \alpha_{-}}} \text {and } \frac{A_{+} e^{7 i \alpha_{+}}+A_{-} e^{7 i \alpha_{-}}}{A_{+} e^{-i \alpha_{+}}+A_{-} e^{-i \alpha_{-}}},
$$

respectively.

The monodromies of the components $e^{\mp i \omega x}$ are given by Eqs. (51,52). In order to compute the monodromy contribution from contour $I$, we notice that the contour passes close to the horizon and the cosmological horizon. There, the mode functions are purely ingoing and outgoing functions, $e^{i \omega x}$ and $e^{-i \omega x}$ respectively. Therefore, the monodromy of the mode function is the monodromy of $e^{i \omega x}$ at the horizon and that of $e^{-i \omega x}$ at the cosmological horizon. The total monodromy is

$$
\begin{gathered}
\text { asymptotic de Sitter }: \exp \left[\pi \omega\left(\frac{1}{\kappa_{h}}-\frac{1}{\kappa_{c}}\right)\right] \\
\text { asymptotic flat }: \exp \left[\frac{\pi \omega}{\kappa_{h}}\right] .
\end{gathered}
$$

Substituting expressions $(51,52,59,60,61)$ in Eq. (49), we get, for asymptotic de Sitter spacetimes

$$
\begin{gathered}
\frac{A_{+} e^{5 i \alpha_{+}}+A_{-} e^{5 i \alpha_{-}}}{A_{+} e^{i \alpha_{+}}+A_{-} e^{i \alpha_{-}}}=e^{\frac{2 \pi \omega}{\kappa_{h}}}, \\
\frac{A_{+} e^{7 i \alpha_{+}}+A_{-} e^{7 i \alpha_{-}}}{A_{+} e^{-i \alpha_{+}}+A_{-} e^{-i \alpha_{-}}}=e^{-\frac{2 \pi \omega}{\kappa_{c}}} .
\end{gathered}
$$

[For completeness, we have given the detailed derivation of the above result in Appendix (B).]

In order to obtain the asymptotically flat results, we set $\kappa_{c} \rightarrow 0^{-}$. Eqn. (62a) is independent of $\kappa_{c}$ and remains unaffected. The RHS of (62b) either grows or decays exponentially depending on whether $\Re(\omega)$ is positive or negative. Thus, for the asymptotically flat case, we obtain

$$
\begin{aligned}
& \frac{A_{+} e^{5 i \alpha_{+}}+A_{-} e^{5 i \alpha_{-}}}{A_{+} e^{i \alpha_{+}}+A_{-} e^{i \alpha_{-}}}=e^{\frac{2 \pi \omega}{\kappa_{h}}}, \\
& A_{+} e^{-i \alpha_{+}}+A_{-} e^{-i \alpha_{-}}=0(\Re(\omega)>0), \\
& A_{+} e^{7 i \alpha_{+}}+A_{-} e^{7 i \alpha_{-}}=0(\Re(\omega)<0) .
\end{aligned}
$$

Note that the constraint (63b) is same as that obtained in Ref. [16]. However, the constraint we obtain in Eq. (63c) 
is new. As we will see below, this constraint gives the correct asymptotic QNM frequency with $\Re\left(\omega_{Q N M}\right)<0$. In all the earlier analysis, in order to obtain the second set of asymptotic QNM frequencies $\left(\Re\left(\omega_{Q N M}\right)<0\right)$, the authors run the contour in the opposite direction. In our formalism, this emerges naturally.

Eliminating $A_{+}, A_{-}$from $(62,63)$, we obtain the expression for the asymptotic QNM frequencies for the two cases:

$$
\tanh \left(\frac{\pi \omega_{Q N M}}{\kappa_{h}}\right) \tanh \left(\frac{\pi \omega_{Q N M}}{\kappa_{c}}\right)=\frac{2}{\tan ^{2}\left[\frac{\pi(q D-2)}{4}\right]-1},
$$

(asymptotic de Sitter spacetimes)

$\frac{2 \pi \omega_{Q N M}}{\kappa_{h}}=(2 n+1) i \pi \pm \log \left[1+2 \cos \left(\frac{\pi(q D-2)}{2}\right)\right]$.

(asymptotically flat spacetimes)

We would like to stress the following points regarding the above results: First, the above results are valid for a single event-horizon, spherically symmetric spacetimes which are asymptotically flat and de Sitter. Second, we have obtained the asymptotic QNM frequencies for the two cases in a unified manner. Third, the expression for the asymptotic de Sitter spacetimes - unlike the flat spacetime - is transcendental, hence it is not possible to obtain solutions uniquely. Fourth, in the case of asymptotic de Sitter spacetimes our result matches with that of Natario and Schiappa [40] for the specific case $q D=2$. Fifth, in the limit of $\kappa_{c} \rightarrow 0^{-}$, Eq. (64) gives the expression for the asymptotically flat spacetimes (65). In the limit of $k_{h} \rightarrow 0^{+}$, Eq. (64) gives

$$
\frac{\omega_{Q N M}}{\kappa_{c}}=-\frac{1}{2 \pi} \ln (3) \pm i\left(\frac{1}{2}+n\right),
$$

which is not the same for the exact de Sitter. As shown earlier [40], the asymptotic de Sitter limit does not provide the correct limit for pure de Sitter. Lastly, in the case of asymptotically flat spacetimes, even though the condition (63c) is different compared to that obtained in the earlier analyses, the expression for the high QNM frequencies are exactly the same.

\section{B. Asymptotic Anti-de Sitter spacetimes}

In this case we can not calculate the monodromy, since the Stokes line do not form a closed contour. Instead, we do the following: We calculate the exact mode function near the generic singularity $\left(B^{\prime}\right)$, horizon $\left(r_{h}\right)$ and spatial infinity $\left(A^{\prime}\right)$. We find the asymptotic limit of the modes along the branches 1 and $1^{\prime}$. [Note that the asymptotic limit corresponding to $\omega x \rightarrow \infty$ corresponds to spatial infinity while $\omega x \rightarrow-\infty$ corresponds to the event horizon.] Matching the asymptotic solutions with the exact solutions at $A^{\prime}$ and $r_{h}$, we obtain the analytic expression for the high QNM frequencies. [We follow the notation of Ref. [40] closely to provide easy comparison.]
First, we match the asymptotic and exact mode function at spatial infinity by going along branch 1 . The exact mode functions at point $B^{\prime}$ is given by Eq. (42). The asymptotic limit corresponding to point $A^{\prime}$ i. e. $\omega x \rightarrow \infty$ is given by Eq. (46).

The exact mode function at spatial infinity $A^{\prime}$ is given by Eq. (A10). Using the relations [cf. Ref. [62]]

$$
\begin{aligned}
J_{n}(z) & \sim\left(\frac{2}{\pi z}\right)^{1 / 2} \cos \left(z-\frac{n \pi}{2}-\frac{\pi}{4}\right) \\
J_{n+1 / 2}(z) & =\left(\frac{2}{\pi}\right)^{1 / 2} z^{n+1 / 2}\left(-\frac{d}{z d z}\right)^{n}\left(\frac{\sin z}{z}\right),
\end{aligned}
$$

(65) (for odd and even dimensions respectively), Eq. (A10) can be rewritten as

$$
R(x)=B_{+}\left[e^{-i \beta_{+}} e^{i \omega x_{0}} e^{-i \omega x}+e^{i \beta_{+}} e^{-i \omega x_{0}} e^{i \omega x}\right],
$$

where

$$
\beta_{+}=\frac{\pi}{4}\left(1+j_{\infty}\right)
$$

Comparing the coefficients of $e^{ \pm i \omega x}$ in the expressions $(46,68)$, we get the first constraint equation:

$$
\frac{A_{+} e^{i \alpha_{+}}+A_{-} e^{i \alpha_{-}}}{A_{+} e^{-i \alpha_{+}}+A_{-} e^{-i \alpha_{-}}}=\frac{e^{-i \beta_{+}} e^{i \omega x_{0}}}{e^{i \beta_{+}} e^{-i \omega x_{0}}} .
$$

Having obtained the first constraint, our next step is to match the exact and asymptotic modes at the horizon by going along the branch $1^{\prime}$. In order to do that, we need to know the exact mode function at $B$. To obtain the mode function $R(x)$ at $B$, we need to deform the contour close to the singularity (in the $r$-plane) by an angle $(-\pi q / 2)$. From $(7)$, this translates to a rotation by an angle $(-\pi q / 2) \times(2 / q)=-\pi$ in the $x$ plane i.e. $x \rightarrow x^{\prime}=e^{i 3 \pi} x$. Using the relation (55), we get

$$
\begin{aligned}
R(x) & \sim A_{+} \sqrt{2 \pi \omega x} \exp \left(-i 3 \alpha_{+}\right) J_{\frac{j}{2}}(\omega x) \\
& +A_{-} \sqrt{2 \pi \omega x} \exp \left(-i 3 \alpha_{-}\right) J_{-\frac{j}{2}}(\omega x)
\end{aligned}
$$

The asymptotic limit of the above mode functions (corresponding to $r_{h}$ ) reduces to the following simple form:

$$
\begin{aligned}
R(x) & \sim\left(A_{+} e^{-3 i \alpha_{+}}+A_{-} e^{-3 i \alpha_{-}}\right) e^{-i \omega x} \\
& +\left(A_{+} e^{-i \alpha_{+}}+A_{-} e^{-i \alpha_{-}}\right) e^{i \omega x} .
\end{aligned}
$$

Comparing the coefficients of $\epsilon^{ \pm i \omega x}$ in the expressions $(33 \mathrm{a}, 72)$, we get the second constraint equation:

$$
A_{+} e^{-3 i \alpha_{+}}+A_{-} e^{-3 i \alpha_{-}}=0 \text {. }
$$

Eliminating $A_{+}, A_{-}$from $(70,73)$, we obtain the analytical expression for the asymptotic QNM frequencies for the asymptotic anti-de Sitter spacetimes:

$$
\omega_{Q N M} x_{0}=\frac{\pi}{2}\left(2 n+\frac{D+3}{2}\right)-\frac{i}{2} \log \left[2 \cos \frac{\pi(q D-2)}{4}\right] .
$$


[For continuity, we have given the detailed derivation of the above result in Appendix (B).]

We would like to stress the following points regarding the above result: First, the above result is valid for a general, single-horizon spherically symmetric asymptotic anti-de Sitter spacetimes. Second, unlike the asymptotic flat spacetime, the high QNM frequencies has no generic features. This is because $x_{0}$ is a arbitrary complex number which depends on the properties of the spacetime. Third, $\omega_{Q N M} x_{0}$ is purely real when $q D=10 / 3$. In the case of $D=2, q=1$, we get

$$
\omega_{Q N M} x_{0}=\left(n+\frac{1}{4}\right) \pi-\frac{i}{2} \log 2
$$

Lastly and more importantly, it is clear from the above expression that the real and imaginary parts of the QNM frequency are of similar order unlike the asymptotic flat/de Sitter cases.

\section{APPLICATION TO SPECIFIC BLACK HOLES}

In the previous section, we obtained master equations for the high frequency QNM for a spherically symmetric black hole with a generic singularity with three different asymptotic properties. As we have shown, the real part of the high frequency QNM is not necessarily proportional to $\ln (3)$ as in the case of $(D+2)$-dimensional Schwarzschild. In order to illustrate this fact, we take specific examples and obtain their QNM.

\section{A. $(D+2)$-dimensional Schwarzschild-de Sitter}

In the case of $(D+2)$-dimensional Schwarzschildde Sitter, the functions $f(r), g(r)$ and $\rho(r)$ in the lineelement (2) are given by

$$
f(r)=g(r)=1-\left(\frac{r_{h}}{r}\right)^{D-1}+\frac{r^{2}}{\ell^{2}} ; \rho(r)=r,
$$

where $r_{h}$ is related to the black hole mass $(M)$ and the $(D+2)$-dimensional cosmological constant $\Lambda$.

Comparing Eqs. (6,76), we get

$$
p=\frac{1-D}{D} ; \quad q=\frac{2}{D} ; \quad x=\frac{r^{D}}{\beta},
$$

Substituting the above expressions Eq. (64), we get

$$
\tanh \left(\pi \omega_{Q N M} / \kappa_{h}\right) \tanh \left(\pi \omega_{Q N M} / \kappa_{c}\right)=-2 .
$$

The above expression matches with that obtained by the previous authors [40].

\section{B. $(D+2)$-dimensional Schwarzschild Anti-de Sitter}

In the case of $(D+2)$-dimensional Schwarzschild antide Sitter, the functions $f(r), g(r)$ and $\rho(r)$ in the lineelement (2) are given by

$$
f(r)=g(r)=1-\left(\frac{r_{h}}{r}\right)^{D-1}-\frac{r^{2}}{\ell^{2}} ; \rho(r)=r,
$$

where $r_{h}$ is related to the black hole mass $(M)$ and the $(D+2)$-dimensional cosmological constant.

Near the singularity, the structure of the metric is same as that of the Schwarzschild-de Sitter. Thus, near the singularity the expressions remain the same [cf. (77)].

Substituting the above expressions Eq. (74), we get

$$
\omega_{Q N M} x_{0}=\frac{\pi}{2}\left(2 n+\frac{D+3}{2}\right)-\frac{i}{2} \log (2) \text {. }
$$

Even though the above expression is valid of Schwarzschild-anti de Sitter black holes it is, in general, not possible to obtain a explicit expression for the $x_{0}$ since it is a complicated function of $M$ and $\ell^{2}$. It is possible to obtain a closed expression of $x_{0}$ only in the large black hole limit (horizon radius $/ \ell \ll 1$ ):

$$
x_{0}=\frac{\pi}{2 \kappa_{h}} \frac{\exp [-i \pi /(D+1)]}{\sin [-i \pi /(D+1)]}
$$

\section{Non-rotating BTZ black hole}

The line-element of the 3-dimensional non-rotating BTZ black hole [64] is

$$
d s^{2}=-\left(\frac{r^{2}}{\ell^{2}}-M\right) d t^{2}+\left(\frac{r^{2}}{\ell^{2}}-M\right)^{-1} d r^{2}+r^{2} d \varphi^{2}
$$

where $M$ is the mass of the black hole and $\ell^{2}$ is related to the negative 3 - $d$ cosmological constant. The above solution has an event horizon at $\ell \sqrt{M}$ while there is no singularity at the origin.

Even though the BTZ line-element does not have a singularity at the origin, it is possible to compare the line-element with that of the generic singularity (5). We get

$$
p=0 ; \quad q=2 ; \quad x \sim r .
$$

Substituting the above expressions in Eq. (74), we get

$$
\omega_{Q N M}=-2 i \sqrt{M}(n+1)+\frac{\log 2}{\pi} .
$$

The real part in the RHS of the above expression does not match with that of the earlier analyses (cf. Ref. [36]). In all the earlier analyses, the real part is equal to $l$ (constant). 
The reason for the discrepancy is as follows: (i) Our analysis, rests on the fact that the spacetime has a singularity at the origin. However, the BTZ black hole does not have a singularity. (ii) For BTZ $p-q+2=0$, hence the second condition in Eq. (24) is violated. This implies that the near the origin, the dominant term in the Regge-Wheeler potential is not given by Eq. (40) and by the following expression:

$$
V[r(x)] \stackrel{r \rightarrow 0}{\sim} \frac{l^{2}}{r^{2}} * M
$$

These suggest that the naive application of our formalism does not work.

\section{DISCUSSION AND CONCLUSION}

In this work, we have computed the high frequency QNMs for scalar perturbations of spherically symmetric single horizons in $(D+2)$-dimensional - asymptotically flat, de Sitter and anti-de Sitter - spacetimes. We have computed these modes using the monodromy approach [16]. In all the three cases, we have shown that the asymptotic frequency of these modes depends on the surface gravity of the event horizon $\left(\kappa_{h}\right)$, the cosmological constant $(\Lambda)$, dimension $(D)$ and the power-law index $(q)$ of $S^{D}$ near the singularity.

Unlike the earlier analyses, we have computed the highfrequency QNMs for the asymptotically flat and de Sitter spacetimes in a unified manner. We have shown that: (i) In the case of asymptotic flat spacetimes, the real part of the high frequency modes has a logarithmic dependence, although the argument of the logarithm is not necessarily an integer. (ii) In the case of asymptotic non-flat spacetimes, the real part of the high-frequency modes, in general, do not have a lograthmic dependence. We have also applied our results to specific examples. In the case of $(D+2)$-dimensional Schwarzschild de Sitter and anti-de Sitter spacetimes, our results match with that of Natario and Schiappa [40]. However, the naive application of our formalism does not work for the non-rotating BTZ black hole. This is due to the fact that the BTZ black hole is non-singular at the center.

The analysis differs from that of the earlier analyzes in two ways: Firstly, using our analyis, a universal feature seems to emerge on the dependence of the high QNM frequencies. It is clear from Eqs. $(64,65,74)$ that the asymptotic QNM frequencies depend on the power-law index $q$ and not $p$. More importantly, the high QNM frequencies seem to have universal dependence of the form $(D q-2) / 2$. Such a feature does not emerge from the previous analyses especially from that of Natario and Schiappa [40]. Secondly, our analysis can be extended to the time-dependent black-holes. In such a case, the generalized Regge-Wheeler potential (23) will be timedepedent. Recently, Xue etal [55] have numerically obtained the QNM frequencies for 4D Schwarzschild. They showed that the QNM frequencies change due to the time-depedence. It will be interesting to analyze their results for the generic spherically symmetric space-times.

In the light of the above results, let us re-examine Hod's conjecture, which rests on the fact that black hole entropy $S_{B H}$ is equispaced and the number of black hole states $\Omega=\exp \left(S_{B H}\right)$ is an integer. Consider the adiabatic invariant:

$$
I=\int \frac{d E}{\omega_{Q N M}} .
$$

In the case of flat spacetimes, it turned out generically that $\omega_{Q N M} \propto T_{H}[\mathbf{I}]$. From this and the first law of black hole thermodynamics, one obtains:

$$
I \propto S_{B H}
$$

where $S_{B H}$ is the black hole entropy. Since adiabatic invariants are supposed to be equispaced, it follows that $S_{B H} \propto n$, an integer. Further, if the proportionality constant is of the form $\ln$ (interger), then $\Omega$ is an integer. In case of asymptotically de Sitter spacetimes however, there is no closed form algebraic expression for the asymptotic QNM frequencies. Thus, it is not clear whether $S_{B H}$ is equispaced, and the degeneracy an integer. For asymptotically anti-de Sitter spacetimes, although $\omega_{Q N M}$ can be expressed in a closed form, the latter depends on the undetermined quantity $x_{0}$. Hence, once again, it seems unlikely that $S_{B H}$ is equispaced and $\Omega$ an integer. Thus, it appears that properties which held for asymptotically flat spacetimes, may no longer hold under more general circumstances.

In order for the asymptotic QNM frequencies to be related to the black hole entropy, the following quantity needs to be an adiabatic invariant (see, for example, Ref. $[10])$ :

$$
I=\int \frac{d E}{\omega_{Q N M}} .
$$

The crucial ingredient in order to show that $I$ is an adiabatic invariant is $\Re\left(\omega_{Q N M}\right) \propto T_{H}$. Although it is straight forward to show that $I$ is indeed an adiabatic invariant in the case of asymptotic flat spacetimes, however it is far from obvious (and in the worst scenario, not true,) for the asymptotic non-flat spacetimes. In the case of asymptotic de Sitter spacetimes since there is no algebraic solution for the asymptotic QNM frequencies, it is not possible to show that $I$ is an adiabatic invariant. In the case of asymptotic anti-de Sitter spacetimes, althought the algebraic structure exists the asymptotic modes crucially depend on the form of $x_{0}$ (whose form is not known), hence it is again not possible to show, in general, $I$ is an adiabatic invariant.

Thus, in the case of asymptotically non-flat spacetimes, using high-frequency QNMs it is not possible to confirm Bekenstein's conjecture that horizon area is an adiabatic invariant implying that the Hod's conjecture may be restrictive. 


\section{Acknowledgments}

This work was supported by the Natural Sciences and Engineering Research Council of Canada. We would like to thank V. Cardoso and J. M. Natario for useful e-mail correspondences. AG and SS would like to thank the Department of Physics, University of Lethbridge, Canada for hospitality where most of this work was done.

\section{APPENDIX A: ASYMPTOTIC ADS SOLUTIONS}

In this appendix, we obtain the solution to the ReggeWheeler equation (22) for the asymptotic AdS spacetimes. We follow closely the approach given by Ref. [52]. For these spacetimes, we have $f(r)=g(r)$ and $\rho(r)=r$.

Substituting the Regge-Wheeler potential (29) in Eq. (22), we get,

$$
\begin{aligned}
R(x) & \sim B_{+} \sqrt{2 \pi \omega\left(x_{0}-x\right)} J_{\frac{j_{\infty}}{2}}\left(\omega\left(x_{0}-x\right)\right) \\
& +B_{-} \sqrt{2 \pi \omega\left(x_{0}-x\right)} J_{-\frac{j_{\infty}}{2}}\left(\omega\left(x_{0}-x\right)\right),
\end{aligned}
$$

where $B_{ \pm}$are the constants of integration determined by the boundary conditions and the quantities $J_{\mu}$ are the Bessel functions of order $\mu$. Using the Bessel form for small arguments [cf. Ref. [62], p. 360]

$$
J_{\nu}(x) \sim x^{\nu} \quad \text { as } \quad x \rightarrow 0
$$

we get

$$
\begin{aligned}
& \sqrt{\omega\left(x_{0}-x\right)} J_{-\frac{j_{\infty}}{2}}\left[\omega\left(x_{0}-x\right)\right] \sim\left(x_{0}-x\right)^{-\frac{D}{2}} \rightarrow \infty \\
& \sqrt{\omega\left(x_{0}-x\right)} J_{\frac{j_{\infty}}{2}}\left[\omega\left(x_{0}-x\right)\right] \rightarrow 0 .
\end{aligned}
$$

Thus, in the spatial infinity, one of the mode function blows up while the other decays.

In order to see things more transparently, let us perform a coordinate transformation such that the ReggeWheeler potential remains finite at infinity. Introducing the following transformation:

$$
\tilde{r}=\log \left(r-r_{h}\right), R(r)=\frac{\left(r-r_{h}\right)^{\frac{1}{2}}}{\sqrt{f}} \tilde{R}(\tilde{r}),
$$

we get

$$
\partial_{\tilde{r}}^{2} \tilde{R}(\tilde{r})-\tilde{V}[r(\tilde{r})] \tilde{R}(\tilde{r})=0
$$

where

$$
\begin{aligned}
& \tilde{V}(r)=-\frac{\left(r-r_{h}\right)^{2}}{f^{2}} \omega^{2}+\frac{\left(r-r_{h}\right)^{2}}{f} \frac{l(l+D-1)}{r^{2}} \\
& -\frac{\left(r-r_{h}\right)^{\frac{3}{2}}}{\sqrt{f r^{D}}} \frac{d}{d r}\left[f r^{D} \frac{d}{d r}\left\{\frac{\left(r-r_{h}\right)^{\frac{1}{2}}}{\sqrt{f r^{D}}}\right\}\right]
\end{aligned}
$$

and $r$ is understood to be $r(\tilde{r})$. In the spatial infinity $\left(f(r) \sim|\Lambda| r^{2}\right)$, we get

$$
\tilde{V}(r) \stackrel{r \rightarrow \infty}{\sim} \frac{(D+1)^{2}}{4}
$$

It is easy to note that Regge-Wheeler potential is positive definite at infinity leading to a pair of exponential solutions:

$$
\tilde{R}(\tilde{r}) \sim \exp \left( \pm \frac{D+1}{2} \tilde{r}\right)
$$

Using (A4), we get,

$$
\begin{aligned}
& \frac{R(r)}{r^{\frac{D}{2}}} \stackrel{r \rightarrow \infty}{\sim} r^{\left(-\frac{D+1}{2} \pm \frac{D+1}{2}\right)} \\
& R(r) \stackrel{r \rightarrow \infty}{\sim} C_{1}^{+} r^{\frac{D}{2}}+C_{2}^{+} r^{-\left(\frac{D}{2}+1\right)}
\end{aligned}
$$

The last expression is identical to Eq. (32) in Sec. (III B). $C_{1}^{+}=0$ (which also implies $B_{-}=0$ ) corresponds to the reflection boundary conditions. Thus, the exact mode function at spatial infinity with the reflection boundary condition is given by

$$
R(x) \sim B_{+} \sqrt{2 \pi \omega\left(x_{0}-x\right)} J_{\frac{j \infty}{2}}\left(\omega\left(x_{0}-x\right)\right)
$$

\section{APPENDIX B: CALCULATIONS}

In this appendix, we outline the essential steps leading to the master equations $(65,64,74)$.

\section{Asymptotic flat and de Sitter spacetimes}

Substituting expressions $(51,52,59,60,61)$ in Eq. (49), for the asymptotic de Sitter and asymptotic flat spacetimes, we get,

$$
\begin{aligned}
& \frac{A_{+} e^{5 i \alpha_{+}}+A_{-} e^{5 i \alpha_{-}}}{A_{+} e^{i \alpha_{+}}+A_{-} e^{i \alpha_{-}}} \times e^{-\pi \omega\left(\frac{1}{\kappa_{h}}+\frac{1}{\kappa_{c}}\right)}=e^{\pi \omega\left(\frac{1}{\kappa_{h}}-\frac{1}{\kappa_{c}}\right)} \\
& \frac{A_{+} e^{7 i \alpha_{+}}+A_{-} e^{7 i \alpha_{-}}}{A_{+} e^{-i \alpha_{+}}+A_{-} e^{-i \alpha_{-}}} \times e^{\pi \omega\left(\frac{1}{\kappa_{h}}+\frac{1}{\kappa_{c}}\right)}=e^{\pi \omega\left(\frac{1}{\kappa_{h}}-\frac{1}{\kappa_{c}}\right)}
\end{aligned}
$$

Simplifying the above expressions we get (62).

Eliminating $A_{ \pm}$in Eq. (62), for the asymptotic de Sitter spacetimes, we get 


$$
\left|\begin{array}{cc}
e^{5 i \alpha_{+}}-e^{\frac{2 \pi \omega}{\kappa_{h}}} e^{i \alpha_{+}} & e^{5 i \alpha_{-}}-e^{\frac{2 \pi \omega}{\kappa_{h}}} e^{i \alpha_{-}} \\
e^{7 i \alpha_{+}}-e^{-\frac{2 \pi \omega}{\kappa_{c}}} e^{-i \alpha_{+}} & e^{7 i \alpha_{-}}-e^{-\frac{2 \pi \omega}{\kappa_{c}}} e^{-i \alpha_{-}}
\end{array}\right|=0 \Rightarrow\left|\begin{array}{cc}
e^{-\frac{\pi \omega}{\kappa_{h}}} e^{2 i \alpha_{+}}-e^{\frac{\pi \omega}{\kappa_{h}}} e^{-2 i \alpha_{+}} e^{-\frac{\pi \omega}{\kappa_{h}}} e^{2 i \alpha_{-}}-e^{\frac{\pi \omega}{\kappa_{h}}} e^{-2 i \alpha_{-}} \\
e^{\frac{\pi \omega}{\kappa_{c}}} e^{4 i \alpha_{+}}-e^{-\frac{\pi \omega}{\kappa_{c}}} e^{-4 i \alpha_{+}} e^{\frac{\pi \omega}{\kappa_{c}}} e^{4 i \alpha_{-}}-e^{-\frac{\pi \omega}{\kappa_{c}}} e^{-4 i \alpha_{-}}
\end{array}\right|=0,(\mathrm{~B}
$$

which leads to

$$
\left|\begin{array}{ll}
\sinh \left(\frac{\pi \omega}{\kappa_{h}}-\frac{i \pi}{2}(1+j)\right) & \sinh \left(\frac{\pi \omega}{\kappa_{h}}-\frac{i \pi}{2}(1-j)\right) \\
\sinh \left(\frac{\pi \omega}{\kappa_{c}}+i \pi(1+j)\right) & \sinh \left(\frac{\pi \omega}{\kappa_{c}}+i \pi(1-j)\right)
\end{array}\right|=0 .
$$

Using properties of hyperbolic functions, we obtain the master equation for the asymptotic de Sitter spacetimes (64).

Eliminating $A_{ \pm}$in Eq. (63), for the asymptotic flat spacetimes, we get

$$
\left|\begin{array}{cc}
e^{5 i \alpha_{+}}-e^{\frac{2 \pi \omega}{\kappa_{h}}} e^{i \alpha_{+}} & e^{5 i \alpha_{-}}-e^{\frac{2 \pi \omega}{\kappa_{h}}} e^{i \alpha_{-}} \\
e^{-i \alpha_{+}} & e^{-i \alpha_{-}}
\end{array}\right|=0
$$

$$
\left|\begin{array}{cc}
e^{5 i \alpha_{+}}-e^{\frac{2 \pi \omega}{\kappa_{h}}} e^{i \alpha_{+}} & e^{5 i \alpha_{-}}-e^{\frac{2 \pi \omega}{\kappa_{h}}} e^{i \alpha_{-}} \\
e^{7 i \alpha_{+}} & e^{7 i \alpha_{-}}
\end{array}\right|=0
$$

for the two cases. Simplifying the above expression, we obtain the master equation for the asymptotic flat spacetimes (65).

\section{Asymptotic anti-de Sitter spacetimes}

Eliminating $A_{+}, A_{-}$from $(70,73)$, we get

$\left|\begin{array}{cc}e^{i\left(\alpha_{+}+\beta_{+}-\omega x_{0}\right)}-e^{-i\left(\alpha_{+}+\beta_{+}-\omega x_{0}\right)} & e^{i\left(\alpha_{-}+\beta_{+}-\omega x_{0}\right)}-e^{-i\left(\alpha_{-}+\beta_{+}-\omega x_{0}\right)} \\ e^{-3 i \alpha_{+}} & e^{-3 i \alpha_{-}}\end{array}\right|=0 \Longrightarrow\left|\begin{array}{cc}\sin \left(\alpha_{+}+\beta_{+}-\omega x_{0}\right) & \sin \left(\alpha_{-}+\beta_{+}-\omega x_{0}\right) \\ e^{-3 i \alpha_{+}} & e^{-3 i \alpha_{-}}\end{array}\right|=0$.

Simplifying, we get,

$$
\omega x_{0}=\frac{\pi}{4}+\beta_{+}-i \tanh ^{-1}\left(\frac{\tan \left(\frac{\pi}{4} j\right)}{\tan \left(\frac{3 \pi}{4} j\right)}\right)
$$

Using properties of hyperbolic functions, we get the master equation for the asymptotic anti-de Sitter spacetimes (74).
[1] H.-P. Nollert, Classical and Quantum Gravity 16, 159 (1999).

[2] K. D. Kokkotas and B. G. Schmidt, Living Rev. Rel. 2, 2 (1999), gr-qc/9909058.

[3] S. Kalyana Rama and B. Sathiapalan, Mod. Phys. Lett. A14, 2635 (1999), hep-th/9905219.

[4] G. T. Horowitz and V. E. Hubeny, Phys. Rev. D62, 024027 (2000), hep-th/9909056.

[5] S. Hod, Phys. Rev. Lett. 81, 4293 (1998), gr-qc/9812002.

[6] O. Dreyer, Phys. Rev. Lett. 90, 081301 (2003), grqc/0211076.

[7] H.-P. Nollert, Phys. Rev. D47, 5253 (1993).

[8] J. D. Bekenstein, Lett. Nuovo Cim. 11, 467 (1974).

[9] J. D. Bekenstein and V. F. Mukhanov, Phys. Lett. B360, 7 (1995), gr-qc/9505012.

[10] G. Kunstatter, Phys. Rev. Lett. 90, 161301 (2003), grqc/0212014.

[11] M. Domagala and J. Lewandowski, Class. Quant. Grav. 21, 5233 (2004), gr-qc/0407051.

[12] K. A. Meissner, Class. Quant. Grav. 21, 5245 (2004), gr-qc/0407052.

[13] O. Dreyer, F. Markopoulou, and L. Smolin (2004), hepth/0409056.

[14] S. Alexandrov (2004), gr-qc/0408033.
[15] L. Motl, Adv. Theor. Math. Phys. 6, 1135 (2003), grqc/0212096.

[16] L. Motl and A. Neitzke, Adv. Theor. Math. Phys. 7, 307 (2003), hep-th/0301173.

[17] D. Birmingham, Phys. Lett. B569, 199 (2003), hepth/0306004.

[18] D. Birmingham, S. Carlip, and Y.-j. Chen, Class. Quant. Grav. 20, L239 (2003), hep-th/0305113.

[19] D. Birmingham, I. Sachs, and S. N. Solodukhin, Phys. Rev. Lett. 88, 151301 (2002), hep-th/0112055.

[20] E. Berti, V. Cardoso, and S. Yoshida, Phys. Rev. D69, 124018 (2004), gr-qc/0401052.

[21] A. Maassen van den Brink, J. Math. Phys. 45, 327 (2004), gr-qc/0303095.

[22] S. Musiri and G. Siopsis, Phys. Lett. B563, 102 (2003), hep-th/0301081.

[23] J. Oppenheim, Phys. Rev. D69, 044012 (2004), grqc/0307089.

[24] V. Cardoso, R. Konoplya, and J. P. S. Lemos, Phys. Rev. D68, 044024 (2003), gr-qc/0305037.

[25] V. Cardoso, J. P. S. Lemos, and S. Yoshida, Phys. Rev. D69, 044004 (2004), gr-qc/0309112.

[26] R. A. Konoplya, Phys. Rev. D66, 044009 (2002), hepth/0205142. 
[27] R. A. Konoplya, Phys. Rev. D68, 024018 (2003), grqc/0303052.

[28] M. R. Setare, Class. Quant. Grav. 21, 1453 (2004), hepth/0311221.

[29] R. Konoplya (2004), hep-th/0410057.

[30] M. R. Setare, Phys. Rev. D69, 044016 (2004), hepth/0312061.

[31] T. Tamaki and H. Nomura, Phys. Rev. D70, 044041 (2004), hep-th/0405191.

[32] V. Suneeta, Phys. Rev. D68, 024020 (2003), grqc/0303114.

[33] T. Padmanabhan, Class. Quant. Grav. 21, L1 (2004), gr-qc/0310027.

[34] A. J. M. Medved, D. Martin, and M. Visser, Class. Quant. Grav. 21, 1393 (2004), gr-qc/0310009.

[35] T. R. Choudhury and T. Padmanabhan, Phys. Rev. D69, 064033 (2004), gr-qc/0311064.

[36] V. Cardoso (2004), gr-qc/0404093.

[37] J. Kettner, G. Kunstatter, and A. J. M. Medved (2004), gr-qc/0408042.

[38] S. Das and S. Shankaranarayanan, Class. Quant. Grav. 22, L7 (2005), hep-th/0410209.

[39] V. Cardoso, J. Natario, and R. Schiappa, J. Math. Phys. 45, 4698 (2004), hep-th/0403132.

[40] J. Natario and R. Schiappa (2004), hep-th/0411267.

[41] H. Nomura and T. Tamaki, Phys. Rev. D71, 124033 (2005), hep-th/0504059.

[42] R. G. Daghigh and G. Kunstatter (2005), gr-qc/0505044.

[43] S. Fernando and C. Holbrook (2005), hep-th/0501138.

[44] S. Chakrabarti and K. S. Gupta (2005), hep-th/0506133.

[45] P. Szekeres and V. Iyer, Phys. Rev. D47, 4362 (1993).

[46] M.-N. Celerier and P. Szekeres, Phys. Rev. D65, 123516 (2002), gr-qc/0203094.

[47] M. Blau, M. Borunda, M. O'Loughlin, and G. Papadopoulos, JHEP 07, 068 (2004), hep-th/0403252.
[48] V. Balasubramanian, J. de Boer, and D. Minic, Phys. Rev. D65, 123508 (2002), hep-th/0110108.

[49] A. Ashtekar and A. Magnon, Class. Quant. Grav. 1, L39 (1984).

[50] A. Ashtekar and S. Das, Class. Quant. Grav. 17, L17 (2000), hep-th/9911230.

[51] H. Kodama and A. Ishibashi, Prog. Theor. Phys. 110, 701 (2003), hep-th/0305147.

[52] E. Winstanley, Phys. Rev. D64, 104010 (2001), grqc/0106032.

[53] S. J. Avis, C. J. Isham, and D. Storey, Phys. Rev. D18, 3565 (1978).

[54] V. Cardoso, J. P. S. Lemos, and S. Yoshida, JHEP 12, 041 (2003), hep-th/0311260.

[55] L.-H. Xue, Z.-X. Shen, B. Wang, and R.-K. Su, Mod. Phys. Lett. A19, 239 (2004), gr-qc/0304109.

[56] V. Cardoso and J. P. S. Lemos, Phys. Rev. D64, 084017 (2001), gr-qc/0105103.

[57] E. Berti and K. D. Kokkotas, Phys. Rev. D68, 044027 (2003), hep-th/0303029.

[58] V. Cardoso and J. P. S. Lemos, Phys. Rev. D67, 084020 (2003), gr-qc/0301078.

[59] S. Yoshida and T. Futamase, Phys. Rev. D69, 064025 (2004), gr-qc/0308077.

[60] A. Zhidenko, Class. Quant. Grav. 21, 273 (2004), grqc/0307012.

[61] A. Maassen van den Brink, Phys. Rev. D68, 047501 (2003), gr-qc/0304092.

[62] M. Abramowitz and I. A. Stegun, Handbook of mathematical functions (Dover Publications, New York, 1964).

[63] J. Natario, Private communications (2005).

[64] M. Banados, M. Henneaux, C. Teitelboim, and J. Zanelli, Phys. Rev. D48, 1506 (1993), gr-qc/9302012. 